\title{
Data-Driven Partitioning of Power Networks Via Koopman Mode Analysis
}

\section{$\operatorname{AUTHOR}(S)$ :}

Raak, Fredrik; Susuki, Yoshihiko; Hikihara, Takashi

\section{CITATION:}

Raak, Fredrik ... [et al]. Data-Driven Partitioning of Power Networks Via Koopman Mode Analysis. IEEE Transactions on Power Systems 2016, 31(4): 2799-2808

\section{ISSUE DATE:}

2016-07

URL:

http://hdl.handle.net/2433/245666

\section{RIGHT:}

(C) 2016 IEEE. Personal use of this material is permitted. Permission from IEEE must be obtained for all other uses, in any current or future media, including reprinting/republishing this material for advertising or promotional purposes,

creating new collective works, for resale or redistribution to servers or lists, or reuse of any copyrighted component of this work in other works.; This is not the published version. Please cite only the published version.; この論文は出版社版

でありません。引用の際には出版社版をご確認ご利用ください。 


\title{
Data-Driven Partitioning of Power Networks via Koopman Mode Analysis
}

\author{
Fredrik Raak ${ }^{1}$ a), Yoshihiko Susuki ${ }^{12}$, and Takashi Hikihara ${ }^{1}$
}

\begin{abstract}
This paper applies a new technique for modal decomposition based solely on measurements to test systems and demonstrates the technique's capability for partitioning a power network, which determines the points of separation in an islanding strategy. The mathematical technique is called the Koopman Mode Analysis (KMA) and stems from a spectral analysis of the so-called Koopman operator. Here, KMA is numerically approximated by applying an Arnoldi-like algorithm recently first applied to power system dynamics. In this paper we propose a practical data-driven algorithm incorporating KMA for network partitioning. Comparisons are made with two techniques previously applied for the network partitioning: spectral graph theory which is based on the eigenstructure of the graph Laplacian, and slow-coherency which identifies coherent groups of generators for a specified number of lowfrequency modes. The partitioning results share common features with results obtained with graph theory and slowcoherency-based techniques. The suggested partitioning method is evaluated with two test systems, and similarities between Koopman modes and Laplacian eigenvectors are showed numerically and elaborated theoretically.
\end{abstract}

Index Terms - Power system monitoring, spectral graph theory, power network partitioning, coherency identification.

\section{Notation}

$$
\begin{aligned}
& {[\boldsymbol{x}]_{i}\left(\text { or }[\mathrm{A}]_{i j}\right) \quad i \text {-th (or }(i, j) \text {-th) element of a vector } \boldsymbol{x}} \\
& \text { (or a matrix } \mathrm{A} \text { ) } \\
& \|\boldsymbol{x}\| \quad \text { Euclidean norm of } \boldsymbol{x} \\
& \operatorname{diag}\left(e_{1}, \ldots, e_{n}\right) \quad n \times n \text { diagonal matrix with elements } \\
& \left(e_{1}, \ldots, e_{n}\right) \\
& \top \quad \text { Transpose operation of vectors } \\
& z^{\mathrm{c}} \quad \text { Complex-conjugate of } z \in \mathbb{C} \\
& z=|z| \angle \phi \quad \text { Angle notation of } z \text { with modulus }|z| \\
& \text { and argument } \phi:=\Im[\ln z] \\
& \mathcal{N}(\text { or } \mathcal{K}) \quad \text { Set }\{1, \ldots, N\}(\text { or }\{1, \ldots, K\})
\end{aligned}
$$

\footnotetext{
${ }^{1}$ Department of Electrical Engineering, Kyoto University, Nishikyo, Kyoto 615-8510, Japan

2 JST-CREST, 4-1-8 Honcho, Kawaguchi, Saitama 332-0012, Japan

a) f-raak@dove.kuee.kyoto-u.ac.jp
}

\section{Introduction}

New technologies emerging in the power system such as integrated information systems and micro-grids enable new possibilities for coordinated counter-strategies against cascading failures. A commonly proposed strategy to prevent blackouts and limit fault propagation is the controlled islanding strategy [1-4]. A key part of the controlled islanding strategy is the partitioning problem, i.e. to decide where to separate a power network. In this paper, we propose a new method for power network partitioning based on solely measurements of bus-voltage angles.

The partitioning is based on the premise that a power network consists of tightly connected groups of buses (clusters) loosely connected to each other. Existing methods are classified into two types: (static) graph-based and dynamics-based. For the first one, the network partitioning problem has also attracted interest from a purely graph theoretical perspective. If the network is preferably separated by identifying loosely connected clusters, relevant cutsets can be identified by applying graph methods such as spectral graph theory. For the second one, a vast power network exhibits inter-area oscillations (or modes) that cause multiple groups of generators to oscillate in an anti-phase motion. An in-phase group of generators is called coherent. The notion of coherency is used for determining the partition of a target network.

Numerous papers have outlined partitioning methods. In [3] a strategy based on the concept of slow-coherency [5] is presented where optimal separation points are determined using a search algorithm. The same method is applied in [4] to a test-case similar to the infamous blackout 2003 in North America and it is shown that a controlled islanding strategy can improve the network's fault response. The standard slow-coherency technique is extended to include load-buses in [6] to directly provide a partitioning of the network. In [7] a new strategy is proposed based on so-called ordered binary decision diagram. In [8] a simulation study of the same strategy is given and it is modified and extended to an effective real-time strategy in [9]. Spectral graph theory is an integral part in identifying network partitions in [10] where power flow and generator coherency constraints are included and in [11] where they evaluate graph Laplacians constructed using different weight on graph edges (transmission lines) and utilize a dendrogram to derive partitions.

In this paper, we focus on the partitioning problem of 
power networks by utilizing the Koopman Mode Analysis (KMA) [12-15]. KMA is a nonlinear generalization of standard modal decomposition and is based on the point spectrum of the Koopman operator which provides an infinitedimensional, linear description of nonlinear evolution of dynamics. An algorithm for computing the so-called Koopman Modes (KMs) from observation data is developed in [13] and is used as data-driven performance assessment of power systems [16-18]. The contribution of the current paper is to demonstrate a new power network partitioning method by applying the KMA on sampled dynamics of busvoltage angles. Despite solely based on measurements, two relevant properties are derived from the demonstrated partitioning algorithm. First, graph theoretical properties of a target network are captured. Second, similarities from the well-known slow-coherency theory are identified. It is thus expected that dynamically significant partitions are generated by the proposed technique which is proven to capture versatile features from sampled data without models or simplifications. A preliminary version of this work was published in [19]. This work contains substantial improvements to the proposed method and new simulations that do not appear in [19]. In difference to [19], this paper focuses only on the phase coherency of bus-angles and a practical method is outlined which incorporates $K$-means to identify coherent groups of buses. Furthermore, it is demonstrated that the proposed partitioning method yields similar results when applied to data for different disturbance locations in the test network. Finally, a new comparison with slow coherency is made and a theoretical motivation is given.

The advantage of the method presented in this paper compared with the previously mentioned ones is that ours is basically model-free and relies only on actual data on dynamics in the network following a disturbance to identify a suitable network partitioning. On the other hand, graph theoretical methods or linearization techniques are per default constrained by modeling simplifications and/or potentially require substantial amounts of detailed power grid data and parameters to provide accurate results.

The rest of this paper is organized as follows. Section 2 provides a short theory of KMA and a concise description of fundamental concepts in spectral graph theory. Section 3 presents and explains the main idea of the paper: datadriven partitioning via KMA. Section 4 presents a demonstration of the idea with two benchmark test systems. Conclusions of this paper are given in Section 5 .

\section{Theoretical Backgrounds}

In this section, we summarize theoretical backgrounds for the current development and demonstration: KMA and spectral graph theory.

\subsection{Koopman Mode Analysis}

The following theory is based on [12-17]. The Koopman operator [20] defined for arbitrary dynamical systems is a linear operator with the ability to capture nonlinear phenomena. Here, let us introduce the Koopman operator for power network dynamic data. A power network is thought of as a continuous-time dynamical system evolving on a smooth manifold $M$ : for each state $\boldsymbol{x} \in M$,

$$
\frac{d \boldsymbol{x}}{d t}=\boldsymbol{F}(\boldsymbol{x})
$$

where $\boldsymbol{x}$ is the vector that contains internal variables of the system such as generator angles, and $\boldsymbol{F}$ is a nonlinear vector-valued function defined on $M$ to represent the rule of how $\boldsymbol{x}$ evolves in time. Normally, measurements in a system are acquired under uniform sampling. Thus, it is natural to consider the evolution of a discrete-time system as

$$
\boldsymbol{x}_{k+1}=\boldsymbol{\Phi}^{h}\left(\boldsymbol{x}_{k}\right), \quad h:=t_{k+1}-t_{k},
$$

where $\boldsymbol{\Phi}^{h}$ is the time-invariant map defined by solutions of (1) and $t_{k}$ denotes the time instance at a sample. Let us consider a scalar observable $g: M \rightarrow \mathbb{R}$ from (2). The observable is a mathematical model of measurements of the network dynamics via sensors such as voltage phasors and power flows. The Koopman operator $\mathcal{U}$ belongs to a family of operators that applied on $g$, it maps $g$ to a new function $\mathcal{U} g$ as,

$$
(\mathcal{U} g)(\boldsymbol{x}):=\left(g \circ \boldsymbol{\Phi}^{h}\right)(\boldsymbol{x})=g\left(\boldsymbol{\Phi}^{h}(\boldsymbol{x})\right),
$$

where $g$ is composed with $\boldsymbol{\Phi}^{h}$. The Koopman operator itself is linear and thus it is natural to perform the spectral analysis. Eigenvalues $\lambda_{i} \in \mathbb{C}$ and eigenfunctions $\varphi_{i}: M \rightarrow$ $\mathbb{C}$ of $\mathcal{U}$ are defined as

$$
\mathcal{U} \varphi_{i}=\lambda_{i} \varphi_{i}, \quad i=1,2, \ldots
$$

Next, a spectral representation of measured network dynamics based on the Koopman operator is presented. To encompass the scenario when the network is synchronously measured at multiple locations like in a PMUbased wide area measurement system [21], let $\boldsymbol{g}(\boldsymbol{x}):=$ $\left(g_{1}(\boldsymbol{x}), g_{2}(\boldsymbol{x}), \ldots, g_{m}(\boldsymbol{x})\right)^{\top}: M \rightarrow \mathbb{R}^{m}$ be a vector-valued observable. If all the components of $\boldsymbol{g}$ lies within the span of eigenfunctions $\varphi_{i}$, we can expand $\boldsymbol{g}(\boldsymbol{x})$ in terms of eigenfunctions,

$$
\boldsymbol{g}(\boldsymbol{x})=\sum_{i=1}^{\infty} \varphi_{i}(\boldsymbol{x}) \boldsymbol{v}_{i}
$$

where $\boldsymbol{v}_{i} \in \mathbb{C}^{m}$ are vector-valued coefficients in the expansion and depend on the choice of observable. We call $\lambda_{i}$ the $i$-th Koopman Eigenvalue (KE) and $\boldsymbol{v}_{i}$ the $i$-th Koopman Mode (KM). The time evolution of observable $\boldsymbol{g}\left(\boldsymbol{x}_{k}\right)$ from $\boldsymbol{g}\left(\boldsymbol{x}_{0}\right)$ is thus expanded as follows:

$$
\boldsymbol{g}\left(\boldsymbol{x}_{k}\right)=\sum_{i=1}^{\infty} \varphi_{i}\left(\boldsymbol{x}_{k}\right) \boldsymbol{v}_{i}=\sum_{i=1}^{\infty} \lambda_{i}^{k} \varphi_{i}\left(\boldsymbol{x}_{0}\right) \boldsymbol{v}_{i}
$$


This shows that the possibly nonlinear evolution in measured network dynamics is characterized by the spectrum of the Koopman operator $\mathcal{U}$.

It is shown in [13] that an Arnoldi-type algorithm produces a decomposition of the same type as (6) for a finite series. The input of the algorithm is $N+1$ vectors of the measured network dynamic data under uniform sampling,

$$
\left\{\boldsymbol{g}_{0}, \ldots, \boldsymbol{g}_{N}\right\}
$$

where $\boldsymbol{g}_{k} \in \mathbb{R}^{m}$ is the snapshot at the discrete time $k$, and $m$ the number of measurement locations. The algorithm presents the following finite-series approximation of (6):

$$
\left.\begin{array}{rl}
\boldsymbol{g}_{k} & =\sum_{i=1}^{N} \tilde{\lambda}_{i}^{k} \tilde{\boldsymbol{v}}_{i}, \quad k=0, \ldots, N-1, \\
\boldsymbol{g}_{N} & =\sum_{i=1}^{N} \tilde{\lambda}_{i}^{N} \tilde{\boldsymbol{v}}_{i}+\boldsymbol{r}
\end{array}\right\}
$$

where $\boldsymbol{r} \in \mathbb{R}^{m}$ is a residue representing an approximation error. The $N$ pairs $\left(\tilde{\lambda}_{i}, \tilde{\boldsymbol{v}}_{i}\right)$ are the output of the algorithm and behave similarly to KEs and KMs. We refer to the type of data analysis based on (7) as KMA. Dominant KMs in (7) are identified by sorting on the Growth Rate (GR) $\left|\tilde{\lambda}_{i}\right|$ and norm $\left\|\tilde{\boldsymbol{v}}_{i}\right\|$. GR indicates the damping of a $\mathrm{KM}$ and the norm quantifies its contribution in the measured data. We speculate that a heuristic condition to decide on the number of dominant KMs can be derived by evaluating the ratio between a chosen set of KMs and remaining KMs for the sum (7).

A data-driven coherency identification from the measured data via KMA was first proposed in [16] and was theoretically refined in [14]. For a derived KM $\boldsymbol{v}_{i}$ with $\left[\boldsymbol{v}_{i}\right]_{j}=A_{i j} \angle \alpha_{i j}$ where $i \in \mathcal{N}$ and $j \in\{1, \ldots, m\}$, we call $A_{i j}$ the amplitude factor and $\alpha_{i j}$ the initial phase. A coherent group of buses with respect to a particular KM implies that all the buses in the group show in-phase swings with the (single) frequency determined by the KM. Now consider the $i$-th KM $\boldsymbol{v}_{i}$. For a small positive constant $\epsilon$, if for the two scalar observables $j_{1}$ and $j_{2}$ in $\boldsymbol{g}$, the initial phases satisfy $\left|\alpha_{i j_{1}}-\alpha_{i j_{2}}\right|<\epsilon$, then the two observables are called $(\epsilon-)$ phase-coherent with respect to the $i$-th KM. Related to this, we define the sum of Complex Conjugate (CC) (oscillatory) KEs scaled by their KMs as

$$
\begin{aligned}
{\left[\boldsymbol{S}_{\mathrm{CC}, i}\right]_{j}^{k} } & =\lambda_{i}^{k}\left[\boldsymbol{v}_{i}\right]_{j}+\left(\lambda_{i}^{\mathrm{c}}\right)^{k}\left[\boldsymbol{v}_{i}\right]_{j}^{\mathrm{c}} \\
& =2 A_{i j}\left|\lambda_{i}\right|^{k} \cos \left(\alpha_{i j}+k \phi_{i}\right), \lambda_{i}=\left|\lambda_{i}\right| \angle \phi_{i},
\end{aligned}
$$

where setting $k=0$ (initial time) yields

$$
\left[\boldsymbol{S}_{\mathrm{CC}, i}\right]_{j}=2 A_{i j} \cos \left(\alpha_{i j}\right)
$$

which provides a quantitative measure on how the oscillatory KM pair is excited at each measurement location. The real-valued vector $\boldsymbol{S}_{\mathrm{CC}, i}$ will be referred to as the $i$-th spatial shape of oscillatory KMs in (6).

\subsection{Spectral Graph Theory}

In the rest of this section, we introduce fundamental concepts in graph theory based on [22]. Spectral graph theory studies networks in terms of eigenvalues and eigenvectors of associated matrices. We denote a graph by the tuple $\mathcal{G}=(\mathcal{V}, \mathcal{E}, w)$, where $\mathcal{V}$ is the set of vertices, $\mathcal{E}$ is the set of edges, and $w: \mathcal{E} \rightarrow \mathbb{R}$ is the weight function of each edge (e.g. see Fig. 2). For a power network with $m$ buses and an arbitrary number of transmission lines, $\mathcal{V}$ and $\mathcal{E}$ correspond to the sets of buses labeled by integers from 1 to $m$ and transmission lines, respectively. We use the notation $(i, j)$ for representing the edge connecting between vertices $i$ and $j \in \mathcal{V}=\{1, \ldots, m\}$. The weight function $w$ usually assigns every edge unity $(=1)$, line admittance, or steady power flow on the line [11]. The graph considered is said to be connected if there exists a path between any two vertices. The adjacency matrix A for the graph $\mathcal{G}$ is defined as

$$
[\mathrm{A}]_{i j}:= \begin{cases}w(i, j), & \text { if }(i, j) \in \mathcal{E} \\ 0, & \text { otherwise }\end{cases}
$$

By counting the degree of vertex $i$ as $d_{i}:=\sum_{j=1}^{m}[\mathrm{~A}]_{i j}$, the so-called graph Laplacian $\mathrm{L}$ is defined as the following symmetrical matrix:

$$
\mathrm{L}:=\operatorname{diag}\left(d_{1}, d_{2}, \ldots, d_{m}\right)-\mathrm{A} .
$$

Our current interest lies in analyzing the eigenstructure of this matrix. For a connected graph, $\mathrm{L}$ has simple zero eigenvalue, and all of the other eigenvalues are positive. Below, they are listed based on increasing magnitude, that is, $\lambda_{1}=0<\lambda_{2}<\cdots<\lambda_{m}$.

The eigenstructure of $L$ has been exploited for graph partitioning. In [23] the so-called algebraic connectivity is defined and associated with the pair of the 2nd eigenvalue and associated eigenvector, $\left(\lambda_{2}, \boldsymbol{V}_{2}\right)$, where $\boldsymbol{V}_{2}$ is known as the Fiedler vector. For a connected graph $\mathcal{G}$, the socalled graph bisectioning, which partitions $\mathcal{V}$ into two disjoint sets $\mathcal{V}_{1}$ and $\mathcal{V}_{2}$, can be conducted using $\boldsymbol{V}_{2}$ by the following conditions [24]:

$$
i \in \mathcal{V}_{1} \text { if }\left[\boldsymbol{V}_{2}\right]_{i} \geq 0, \quad i \in \mathcal{V}_{2} \text { if }\left[\boldsymbol{V}_{2}\right]_{i}<0 \text {. }
$$

This method will be used in this paper as a conventional one for comparison and we let $w(i, j)=1$ for all edges.

\section{Proposed Data-Driven Partition- ing Method}

In this paper, we demonstrate how a power network is separated into several disjoints parts by applying KMA to busangle dynamics following a disturbance. First of all, we make two assumptions:

(i) Graph information of a target power network is given.

(ii) Bus-angle dynamics are observed for every bus. 
The network's configuration can be obtained from the state estimator's topology processor [25], and hence (i) is reasonable. Assumption (ii) is based on two reasons. First, within foreseeable future we can expect an extensive deployment of synchronized measurement units. Second, with the dynamic state estimator, full observability is achievable even with a limited amount of PMUs. In fact, placing PMUs at about one third of the total number of buses is generally sufficient to achieve full observability [26]. Until such a system is in place, it is possible to construct a state estimator utilizing both SCADA and PMU measurements [25].

The data-driven partitioning method demonstrated in this paper is outlined below. Its pseudocode is provided in Algorithm 1.

1. Under the stated assumptions (i) and (ii), consider the finite-time data on bus-angle dynamics under uniform sampling, given by

$$
\left\{\boldsymbol{\theta}_{0}, \ldots, \boldsymbol{\theta}_{N}\right\},
$$

where $\boldsymbol{\theta}_{k}=\left(\theta_{0, k}, \ldots, \theta_{m, k}\right)^{\top}$ is the $m$-dimensional snapshot of bus-angles at the $k$-th time instance and the number of available snapshots corresponds to $N+1$ (see Fig. 1(a)). By applying the Arnoldi-like algorithm to the finite-time data, $N$ pairs $\left(\tilde{\lambda}_{i}, \tilde{\boldsymbol{v}}_{i}\right)(i \in \mathcal{N})$ of KEs and KMs are obtained (KoopmanModeAnalysis).

2. A set of dominant $\mathrm{KMs}$, denoted by $\mathcal{N}_{\mathrm{d}} \subset \mathcal{N}$, is identified by arranging them in descending order of GR and norm (DominantKMA).

3. For every pair $\left(\tilde{\lambda}_{i}, \tilde{\boldsymbol{v}}_{i}\right)$ in the dominant KMs, the initial phase vector $\boldsymbol{\alpha}_{i}:=\left(\alpha_{i 1}, \ldots, \alpha_{i m}\right)^{\top}$ is calculated (InitialPhaseVector).

4. By plotting all the components of $\boldsymbol{\alpha}_{i}$ on the unit circle, the phase coherency in bus-angles is detected. Below, we use the same notation $\alpha_{i j}$ to represent its plotted point. When the $K$-means clustering algorithm [27] is applied to the $m$ points on the unit circle, $K$ center points called centroids $C_{i}^{\ell}(\ell \in \mathcal{K})$ and associated clusters (subsets of buses) $\mathcal{A}_{i}^{\ell}$ are located (KmeansClustering).

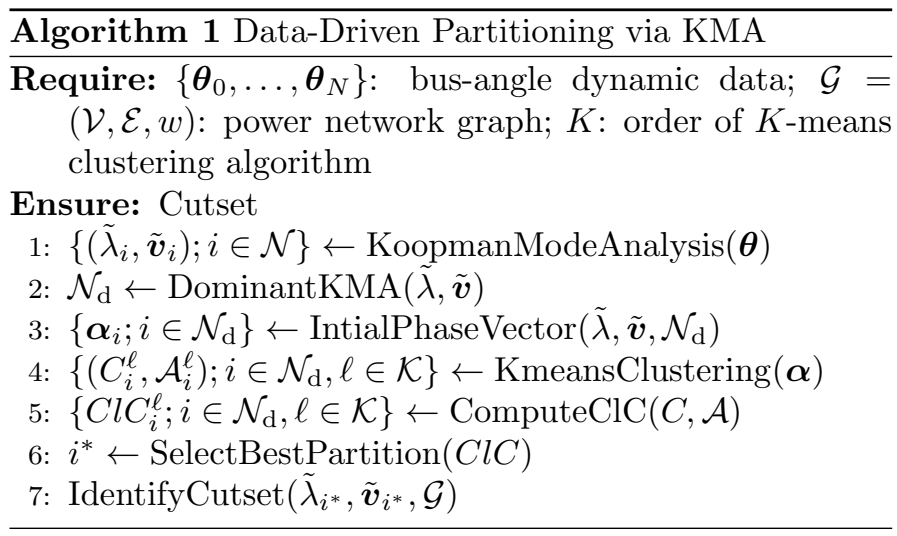

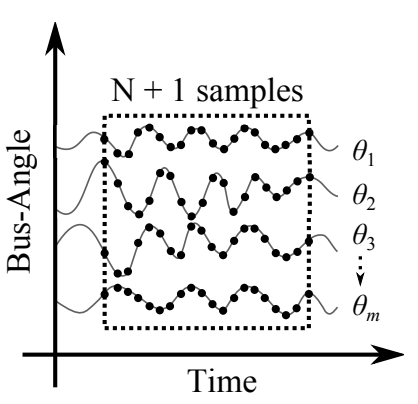

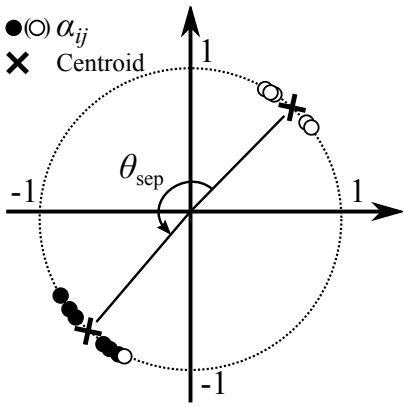

(a) Finite-time, sampled data on (b) Phase coherency in one KM. bus-angles.

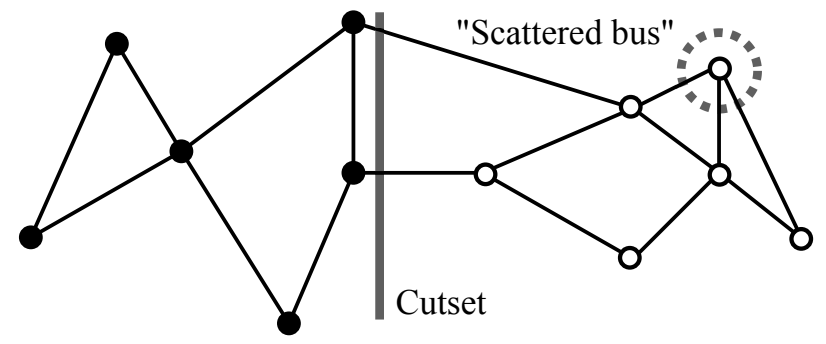

(c) Identified cutset.

Figure 1: Illustration of data-driven network partitioning via Koopman mode analysis described in Algorithm 1.

5. For each $\mathcal{A}_{i}^{\ell}$, the distance between a contained point $\alpha_{i j}$ and its centroid $C_{i}^{\ell}$ is denoted by $\operatorname{dist}\left(\alpha_{i j}, C_{i}^{\ell}\right)$ with the standard Euclidean norm. Thus, we compute the Cluster Coefficient $\mathrm{ClC}$ for each cluster $\mathcal{A}_{i}^{\ell}$ as

$$
C l C_{i}^{\ell}:=\frac{\sum_{j \in \mathcal{A}_{i}^{\ell}} \operatorname{dist}\left(\alpha_{i j}, C_{i}^{\ell}\right)}{\#\left(\mathcal{A}_{i}^{\ell}\right)}
$$

where $\#\left(\mathcal{A}_{i}^{\ell}\right)$ stands for the number of elements belonging to $\mathcal{A}_{i}^{\ell}$ (ComputeClC). A small $\mathrm{ClC}$ indicates a tightly clustered group of buses, and is deemed "small" in comparison to other dominant KMs and previous data.

6. The number of dominant KMs is now decreased from $\#\left(\mathcal{N}_{\mathrm{d}}\right)$ such that only KMs with small ClCs and clear phase separation $\theta_{\text {sep }}$ between centroids are included (see Fig. 1(b); SelectBestPartition).

7. Since from assumption (i) the complete network structure is given, the cutsets are identified by first assigning an index to each bus that represents one of the $K$ groups and second identify the lines connecting buses of different groups (IdentifyCutset). In the case that a singular bus or few buses are detected incoherent with surrounding buses (see Fig.1(c)), the so-called scattered buses may be discarded depending on the preferred partitioning setting. 

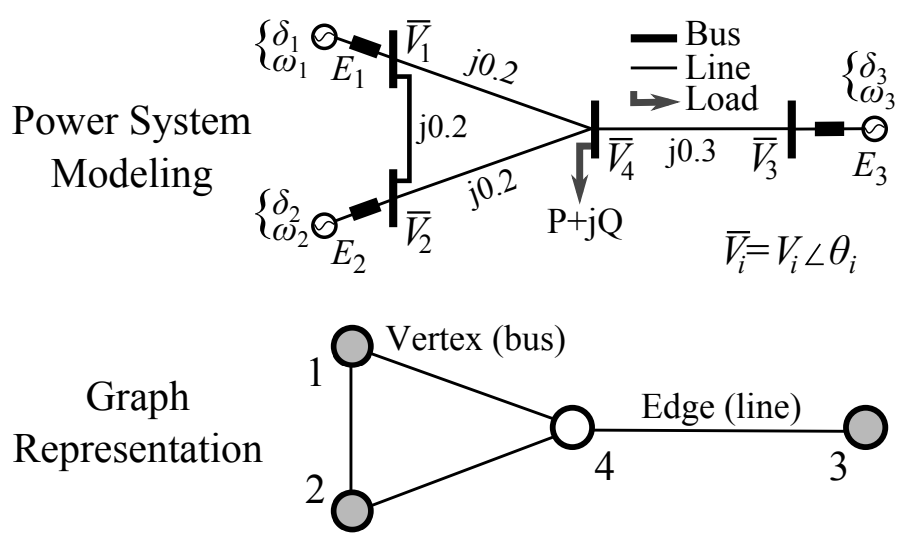

Figure 2: Simple test system used in simulations with three generator buses and one load bus. Two representations are shown; the upper figure depicts typical power system modeling and the lower figure depicts a graph representation in terms of vertices comprised by generator buses (filled circles) and one load bus (hollow circle) connected by edges (transmission lines).

\section{Demonstration}

In this section, we demonstrate the data-driven partitioning method in both simple and more realistic benchmark systems. The details of how to apply the method to dynamic data are described here. A connection between partitioning results obtained with KMA and spectral graph theory is also analytically revealed.

\subsection{Benchmark Systems and Simulation Models}

We use two benchmark systems in this section. The number of buses for each system is denoted as $m$ and the number of generators as $n$. The first is depicted in Fig. 2 and is a simple four-bus test system consisting of three generator buses and one load bus. The second is the IEEE 118bus test system [28] depicted in Fig. 6 and simulated with 19 generators with parameters chosen same as [8]. System equations are solved using the Power System Analysis Toolbox (PSAT) [29] for MATLAB. In PSAT, dynamic responses of the systems are simulated with the following set of nonlinear Differential Algebraic Equations (DAEs):

$$
\frac{d \boldsymbol{x}}{d t}=\boldsymbol{f}(\boldsymbol{x}, \boldsymbol{y}), \quad \mathbf{0}=\boldsymbol{g}(\boldsymbol{x}, \boldsymbol{y}),
$$

where $\boldsymbol{x}$ is the set of state variables consisting of phase angles $\delta_{j}$ and rotor speed deviations $\omega_{j}$ of generators, and $\boldsymbol{y}$ the set of algebraic variables of bus-voltages $V_{j}$ and busangles $\theta_{j}$. The vector-valued function $\boldsymbol{f}$ represents the rule of time-evolution of $\boldsymbol{x}$ defined by the classical swing equations for synchronous generators, and $\boldsymbol{g}$ governs the active and reactive power balance at each bus.

The setting for numerical simulations is summarized below. Throughout the simulations, the sampling frequency $f_{\mathrm{s}}=60 \mathrm{~Hz}$ was used. Bus-voltage angles $\boldsymbol{\theta}$ were sampled and observed from the so-called mean-angle, which is similar to the Center-Of-Inertia (COI) [30] and is defined as follows: for every time $k$,

$$
\bar{\theta}_{k}:=\frac{1}{m} \sum_{j=1}^{m} \theta_{j, k} .
$$

According to simulations, this produces a moving reference similar to the COI reference frame for generator rotor angles. Consequently, a change of reference frame from the proposed one to COI yields similar results on partitioning. Also, by using the proposed reference frame no additional measurement points at generator locations is required.

\subsection{Simple Test System}

We introduce our approach by first considering a small test system which comprises three generator buses and one load bus: see Fig. 2. Parameters of the test system are chosen as follows: base power $S_{\mathrm{b}}$ and generator ratings $S_{\mathrm{g}}$ are set to $100 \mathrm{MVA}$, nominal frequency $f_{\mathrm{n}}=60 \mathrm{~Hz}$ and a constant impedance load $S_{\mathrm{L}}=P_{\mathrm{L}}+\mathrm{j} Q_{\mathrm{L}}=3+\mathrm{j} 0.05$ p.u. Generator parameters are chosen in a simple manner: transient reactances $x_{\mathrm{d}, i}^{\prime}=0.2 \mathrm{p} . \mathrm{u}$., inertia constants $M_{i}=7 \mathrm{~s}$, damping $D_{i}=0.1$, for all generators and mechanical power $P_{\mathrm{m}}=\left\{P_{\mathrm{m}, 1}, P_{\mathrm{m}, 2}, P_{\mathrm{m}, 3}\right\}=\{1.5,0.5,1\}$ p.u. Two complex conjugate oscillatory mode pairs are identified from linearization of system equations (13): $1.64 \mathrm{~Hz}$ and $2.27 \mathrm{~Hz}$.

A partition of the simple test system is presented by KMA of sampled data on bus-angle dynamics. For initial generator frequencies $\omega_{i}^{0}$, a perturbation is initiated as $\omega_{i}^{0}+\Delta \omega_{i}, i=2,3$ with $\Delta \omega_{i}=0.005$ p.u., and the resulting dynamics are shown in Fig. 3. KMA is applied to $5 \mathrm{~s}$ of disturbance data to give $300 \mathrm{KMs}$. Two oscillatory KMs with frequencies $1.66 \mathrm{~Hz}$ and $2.24 \mathrm{~Hz}$, respectively, are identified as dominant in the decomposition. Note that the frequencies of the identified KMs are almost identical to the linear modes. Their spatial shapes denoted by $\boldsymbol{S}_{\mathrm{CC}(1.66 \mathrm{~Hz})}$ and $\boldsymbol{S}_{\mathrm{CC}(2.24 \mathrm{~Hz})}$ are displayed in Fig. 4 together with $\boldsymbol{V}_{2}$ and $\boldsymbol{V}_{3}$, derived from the graph Laplacian of the test system. The spatial shapes $\boldsymbol{S}_{\mathrm{CC}}$ are scaled with appropriate factors for the sake of comparison. The values of elements quantify the connectivity. For $\boldsymbol{S}_{\mathrm{CC}(1.66 \mathrm{~Hz})}$, buses 1 and 2 are strongly connected (coherent) and separated from bus 3. By inspecting the initial phases of $\boldsymbol{v}_{1.66 \mathrm{~Hz}}$ in Fig. 5 it is concluded that bus 4 is also coherent with buses 1 and 2. Thus, the partition according to the $1.66 \mathrm{~Hz} \mathrm{KM}$ leads to two groups of buses: $\{1,2,4\}$ and $\{3\}$. This is intuitive by inspection of the network's structure. The bus-number dependence of elements are close between $\boldsymbol{S}_{\mathrm{CC}(1.66 \mathrm{~Hz})}$ (or $\left.\boldsymbol{S}_{\mathrm{CC}(2.24 \mathrm{~Hz})}\right)$ and $\boldsymbol{V}_{2}\left(\right.$ or $\left.\boldsymbol{V}_{3}\right)$. From this observation, we speculate that the spectral property of the network is extracted from sampled dynamics of bus-angles via spectrum of the Koopman operator. 


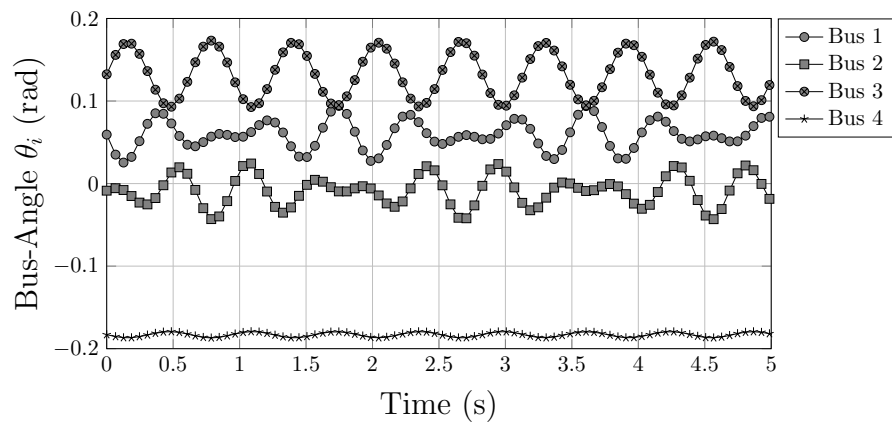

Figure 3: Dynamics of bus-angles $\theta_{i}$ for the simple test system following a small disturbance of initial generator speed $\omega_{i}^{0}$ for $i=2,3$.
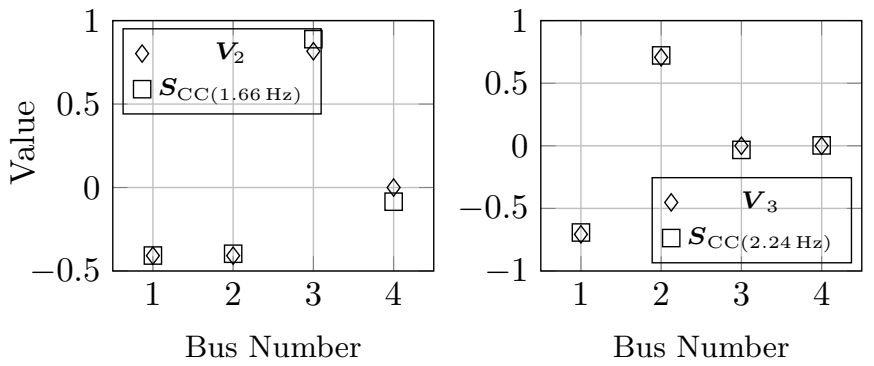

Figure 4: Comparison between eigenvectors $\boldsymbol{V}_{2}, \boldsymbol{V}_{3}$ of the unweighted Laplacian, and spatial shapes $\boldsymbol{S}_{\mathrm{CC}(1.66 \mathrm{~Hz})}$, $\boldsymbol{S}_{\mathrm{CC}(2.24 \mathrm{~Hz})}$ of the dominant KMs identified from dynamics shown in Fig. 3.
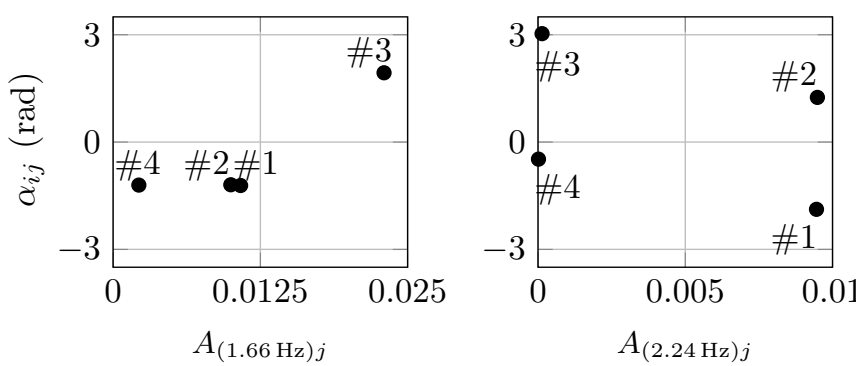

Figure 5: Initial phase $\alpha_{i j}$ vs. amplitude factor $A_{i j}$ plots for $\boldsymbol{v}_{1.66 \mathrm{~Hz}}$ and $\boldsymbol{v}_{2.24 \mathrm{~Hz}}$, where $\# i$ beside a point denotes the bus number.

\subsection{Analytical Evaluation}

The previous section numerically showed a prominent similarity between the Laplacian eigenvectors and spatial shapes of Koopman modes and hence in the dynamics of simple test system. Here, we theoretically clarify the connection between KMA and spectral graph theory in terms of bus-angle dynamics in a general power network model.

Now we introduce the equations of motion for a general power network. Let us denote by $\mathcal{V}_{\mathrm{G}}$ the set of buses with synchronous generators and by $\mathcal{V}_{\mathrm{L}}$ the set of buses with no generator. For a generator connected to bus $i \in \mathcal{V}_{\mathrm{G}}$, the voltage behind reactance is denoted by $E_{i} \angle \delta_{i}$, and the bus- voltage by $V_{i} \angle \theta_{i}$. The differential equations in the DAE model (13) are as follows: for $i \in \mathcal{V}_{\mathrm{G}}$,

$$
\frac{d \delta_{i}}{d t}=\omega_{i}, \quad M_{i} \frac{d \omega_{i}}{d t}=P_{\mathrm{m}, i}-P_{\mathrm{e}, i}-D_{i} \omega_{i},
$$

with the electrical output power $P_{\mathrm{e}, i}$ given by

$$
P_{\mathrm{e}, i}=\frac{E_{i} V_{i}}{x_{\mathrm{d}, i}^{\prime}} \sin \left(\delta_{i}-\theta_{i}\right),
$$

where $x_{\mathrm{d}, i}^{\prime}$ is the transient reactance. According to the standard argument of short-term rotor stability [30], constant voltages and active/reactive power decoupling are assumed. The algebraic variables $\boldsymbol{y}$ coincide with the busangles $\boldsymbol{\theta}$. Thus, the active power part of $\boldsymbol{g}$ is considered: for $i \in \mathcal{V}=\mathcal{V}_{\mathrm{G}} \cup \mathcal{V}_{\mathrm{L}}$,

$$
0=\sum_{j \in \mathcal{V} \backslash\{i\}} V_{i} V_{j}\left|Y_{i j}\right| \cos \left(\theta_{j}-\theta_{i}+\phi_{i j}\right)-P_{i}\left(\boldsymbol{x}, \theta_{i}\right),
$$

where $\left|Y_{i j}\right| \angle \phi_{i j}$ is the admittance of lines connecting bus $i$ and $j$ (if no line exists, we regard its modulo as zero) and $P_{i}\left(\boldsymbol{x}, \theta_{i}\right)$ represents injected power $\left(P_{\mathrm{e}, i}\right)$ for $i \in \mathcal{V}_{\mathrm{G}}$ or constant consumed power $\left(P_{\mathrm{L}, i}\right)$ for $i \in \mathcal{V}_{\mathrm{L}}$. Now, following [31], we use the singular perturbation technique for (13) and introduce a sufficiently small, positive parameter $\epsilon$ as follows:

$$
\frac{d \boldsymbol{x}}{d t}=\boldsymbol{f}(\boldsymbol{x}, \boldsymbol{\theta}), \quad \epsilon \frac{d \boldsymbol{\theta}}{d t}=\boldsymbol{g}(\boldsymbol{x}, \boldsymbol{\theta}) .
$$

By introducing the new independent variable $\tau:=t / \epsilon$, we have

$$
\frac{d \boldsymbol{x}}{d \tau}=\epsilon \boldsymbol{f}(\boldsymbol{x}, \boldsymbol{\theta}), \quad \frac{d \boldsymbol{\theta}}{d \tau}=\boldsymbol{g}(\boldsymbol{x}, \boldsymbol{\theta}) .
$$

By taking the $\epsilon \rightarrow 0$ limit, the boundary layer system is constructed as

$$
\frac{d \boldsymbol{\theta}}{d \tau}=\boldsymbol{g}(\boldsymbol{x}, \boldsymbol{\theta}), \quad \boldsymbol{x} \text { fixed },
$$

which captures the dynamical feature of (fast) bus-angle dynamics in an analytical, self-consistent manner. Here, by linearizing (20) around a feasible equilibrium point $\left(\boldsymbol{x}^{*}, \boldsymbol{\theta}^{*}\right)$ of the DAE system (13), we derive

$$
\frac{d \boldsymbol{\theta}}{d \tau}=\left(\mathrm{L}_{P, \theta}+\mathrm{K}_{P_{\mathrm{e}}, \theta}\right) \Delta \boldsymbol{\theta},
$$

with $\mathrm{L}_{P, \theta}$ defined as

$\left[\mathrm{L}_{P, \theta}\right]_{i, j}:=\left\{\begin{array}{c}\sum_{\ell \in \mathcal{V} \backslash\{i\}} V_{i} V_{\ell}\left|Y_{i \ell}\right| \sin \left(\theta_{\ell}^{*}-\theta_{i}^{*}+\phi_{i \ell}\right), i=j \\ \quad-V_{i} V_{j}\left|Y_{i j}\right| \sin \left(\theta_{j}^{*}-\theta_{i}^{*}+\phi_{i j}\right), i \neq j,\end{array}\right.$

where $\mathrm{L}_{P, \theta}$ is a graph Laplacian matrix of linearized power flow for every bus where the state variables $(\boldsymbol{x})$ are viewed

An equilibrium point $\left(\boldsymbol{x}^{*}, \boldsymbol{\theta}^{*}\right)$ of the DAE system (13) is said to be feasible if the Jacobian matrix of $\boldsymbol{g}$ with respect to $\boldsymbol{y}$ is regular inside a neighborhood of $\left(\boldsymbol{x}^{*}, \boldsymbol{\theta}^{*}\right)$. 


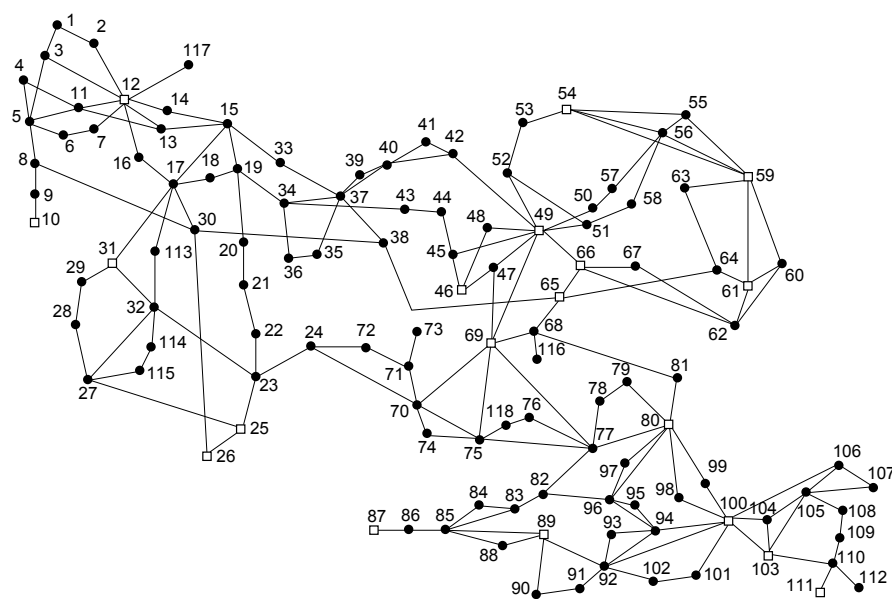

Figure 6: IEEE 118-bus test system [28]. The dynamics of the system are simulated with the classical model of 19 generators. Generator buses are indicated by hollow squares, and filled circles represent load and intermediate buses.

as constant. The matrix $\mathrm{K}_{P_{\mathrm{e}}, \theta}$ is a diagonal matrix and represents the linearized injected power from generators given as follows:

$$
\left[\mathrm{K}_{P_{e}, \theta}\right]_{i, i}:=\left\{\begin{array}{r}
\frac{E_{i} V_{i}}{x_{\mathrm{d}, i}^{\prime}} \cos \left(\delta_{i}^{*}-\theta_{i}^{*}\right), i \in \mathcal{V}_{\mathrm{G}}, \\
0, i \in \mathcal{V}_{\mathrm{L}},
\end{array}\right.
$$

which can be viewed as a perturbation to $\mathrm{L}_{P, \theta}$. The magnitude of perturbation becomes small in particular if the number of buses is much larger than the number of generators. Equation (21) determines the behavior of bus-angle dynamics close to the equilibrium point and is influenced by the graph Laplacian $\mathrm{L}_{P, \theta}$. For the data-driven partitioning method based on bus-angle dynamics proposed in this paper, it makes sense to compare the approach based on the Koopman operator with the network analysis based on spectral graph theory.

\subsection{IEEE 118-bus Test System}

Next, we apply the data-driven partitioning method to a larger test system, the IEEE 118-bus test system (see Fig. 6). For gaining finite-time data on bus-angle dynamics, the system is perturbed by three-phase-faults according to following two cases:

(i) Fault applied to bus 17 with a clearing time $t_{\mathrm{c}}=$ $280 \mathrm{~ms}$, slightly below the critical clearing time $t_{\mathrm{cc}}$.

(ii) Fault applied to bus 100 with a clearing time $t_{\mathrm{c}}=$ $180 \mathrm{~ms}$, slightly below its $t_{\mathrm{cc}}$.

Load flow parameters used are from [28] and generator parameters are identical to [8]. The dynamic responses of all bus-angles for the two cases (i) and (ii) are shown in Fig. 7. For each case, KMA is applied to the post-fault data on bus-angles during $[1 \mathrm{~s}, 8 \mathrm{~s}]$ and provides $420 \mathrm{KMs}$.

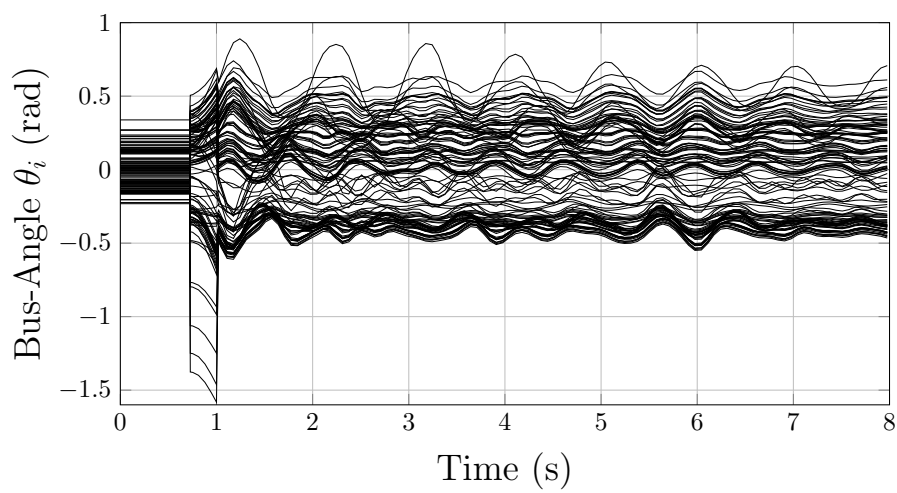

(a) Fault at bus 17 (Case (i)).

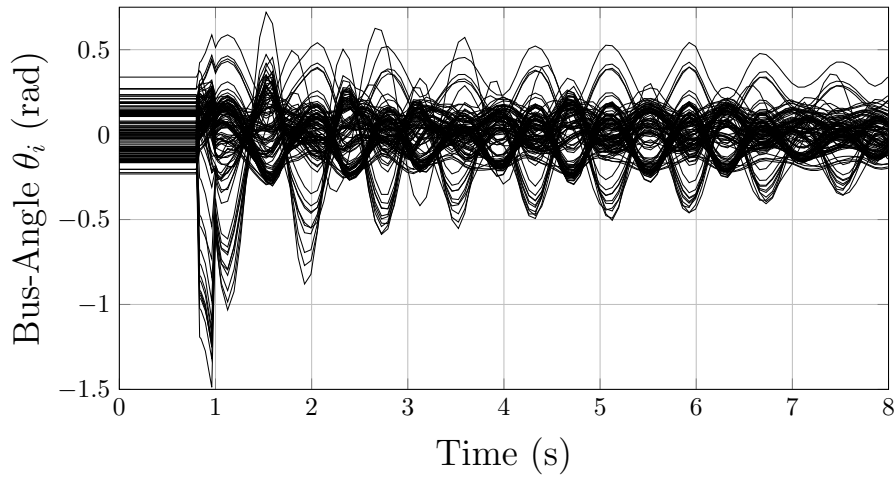

(b) Fault at bus 100 (Case (ii)).

Figure 7: Dynamics of bus-angles $\theta_{i}$ in the IEEE 118-bus test system for (a) fault at bus 17 (Case (i)) and (b) fault at bus 100 (Case (ii)).

Dominant KMs identified in the decompositions are listed in Table 1. The $0 \mathrm{~Hz}$ KMs with unit GR appear for both cases and correspond to the time-averaged values of the bus-angle measurements in the system. Three KM pairs for each fault case are chosen and used for partitioning the system. The chosen KMs have large norms among the dominant oscillatory $\mathrm{KMs}$ and all show small $\mathrm{ClCs}$ and clear separation $\theta_{\text {sep }}$. Initial phase vectors for chosen KMs for both disturbance cases are depicted in Fig. 8. For Case (i), KM-3 $(1.24 \mathrm{~Hz})$ clearly displays 3 large groups of buses, whereas remaining KMs for both the disturbances predominantly contain 2 large groups of buses in anti-phase. Algorithmically speaking, this is identified by applying the partitioning algorithm with first $K=2$ and then $K=3$ and note that the obtained ClCs decrease for the case with $K=3$ while a clear $\theta_{\text {sep }}$ is maintained. Thus for the KM-3, 3 -means clustering is applied, otherwise the 2-means one is used. According to the algorithm, dominant KMs provide partitioning of the system into two or more sub-systems (depending on cohesiveness of coherent buses). Partitions for the chosen dominant KMs based on phase coherency are given in Figs. 9 and 10. For Case (i), KM-3 provides a partition that shares the cutset obtained from spectral graph bisectioning but contains additional cutsets. KM-2 for both the disturbance cases are almost identical in terms of provided partitioning and frequency. A comparison be- 
Table 1: Dominant Koopman modes obtained for the data on voltage angle dynamics shown in Fig. 7. Rows marked in gray indicate KMs that have been chosen for the network partitioning.

\begin{tabular}{|c|c|c|c|c|c|c|}
\hline $\begin{array}{c}\text { Case } \\
\#\end{array}$ & $\begin{array}{c}\text { No. } \\
j\end{array}$ & $\begin{array}{l}\mathrm{GR} \\
\left|\tilde{\lambda}_{j}\right|\end{array}$ & $\begin{array}{c}\text { Freq. }[\mathrm{Hz}] \\
\operatorname{Im}\left[\ln \tilde{\lambda}_{j}\right] /\left(2 \pi T_{s}\right)\end{array}$ & $\begin{array}{l}\text { Norm } \\
\left\|\tilde{\boldsymbol{v}}_{j}\right\|\end{array}$ & $\begin{array}{c}\mathrm{ClC} \\
\mathrm{ClC}_{j}^{\ell}\end{array}$ & $\begin{array}{l}\theta_{\text {sep }} \\
(\operatorname{deg})\end{array}$ \\
\hline & 1 & 1 & 0 & 3.29 & $\begin{array}{l}0 \\
0\end{array}$ & 180 \\
\hline & 2 & 0.9977 & \pm 1.04 & 0.28 & $\begin{array}{l}0.009 \\
0.008\end{array}$ & 177 \\
\hline & 3 & 0.9976 & \pm 1.24 & 0.25 & $\begin{array}{l}0.036 \\
0.018 \\
0.028\end{array}$ & $\begin{array}{l}77 \\
103 \\
180\end{array}$ \\
\hline \multirow[t]{8}{*}{ (i) } & i & 0.9975 & \pm 3.29 & 0.05 & $\begin{array}{l}0.130 \\
0.074\end{array}$ & 173 \\
\hline & $i$ & 0.9970 & \pm 0.21 & 0.02 & $\begin{array}{l}0.091 \\
0.004\end{array}$ & 185 \\
\hline & $i$ & 0.9965 & \pm 2.59 & 0.03 & $\begin{array}{l}0.164, \\
0.246\end{array}$ & 168 \\
\hline & $i$ & 0.9959 & \pm 2.37 & 0.16 & $\begin{array}{l}0.081 \\
0.055\end{array}$ & 179 \\
\hline & 4 & 0.9950 & \pm 2.02 & 0.19 & $\begin{array}{l}0.039 \\
0.043\end{array}$ & 180 \\
\hline & 1 & 1 & 0 & 1.02 & $\begin{array}{l}0 \\
0\end{array}$ & 180 \\
\hline & 2 & 0.9992 & \pm 1.00 & 0.17 & $\begin{array}{l}0.042, \\
0.104\end{array}$ & 167 \\
\hline & 3 & 0.9976 & \pm 1.40 & 0.33 & $\begin{array}{l}0.007, \\
0.044\end{array}$ & 178 \\
\hline \multirow{5}{*}{ (ii) } & i & 0.9970 & \pm 0.83 & 0.1 & $\begin{array}{l}0.079 \\
0.086\end{array}$ & 173 \\
\hline & 1 & 0.9970 & \pm 1.59 & 0.1 & $\begin{array}{l}0.128 \\
0.049\end{array}$ & 176 \\
\hline & 4 & 0.9966 & \pm 1.23 & 1.73 & $\begin{array}{l}0.007 \\
0.004\end{array}$ & 180 \\
\hline & i & 0.9966 & 0 & 0.27 & $\begin{array}{l}0 \\
0\end{array}$ & 180 \\
\hline & i & 0.9965 & 2.46 & 0.04 & $\begin{array}{l}0.016, \\
0.011\end{array}$ & 179 \\
\hline
\end{tabular}

tween the Laplacian eigenvector $\boldsymbol{V}_{2}$ and the spatial shapes $\boldsymbol{S}_{\mathrm{CC}(1.04 \mathrm{~Hz})}$ and $\boldsymbol{S}_{\mathrm{CC}(1.00 \mathrm{~Hz})}$ of KM-2 is given in Fig. 11 (spatial shapes for buses 86 and 87 are not shown due to large magnitudes compared to other buses). It is clear that the same mode is captured for both the disturbance cases and that its shape is similar to $\boldsymbol{V}_{2}$. KM-4 for Case (i) provides a cutset similar to cutsets for KM-3 and 4 for Case (ii).

Here, it should be noted that the partition depends on if buses in a coherent group are geographically adjacent. For instance, it is possible that buses in the north and south of the system are swinging coherently against the central buses for a certain mode. Thus, even though they are detected as coherent in terms of KM, applying 2-means clustering and identifying cutsets will yield a partitioning into 3 groups: see e.g. the broken green circle in Fig. 9 that encircles generator bus 31 which is incoherent with surrounding buses. A special attention should be given to these occurrences.

\subsection{Comparison with Slow-Coherency- Based Partitioning}

As reviewed in Section 1, the slow-coherency-based partitioning method is widely used. Here, for comparison we consider a partition obtained from the method presented
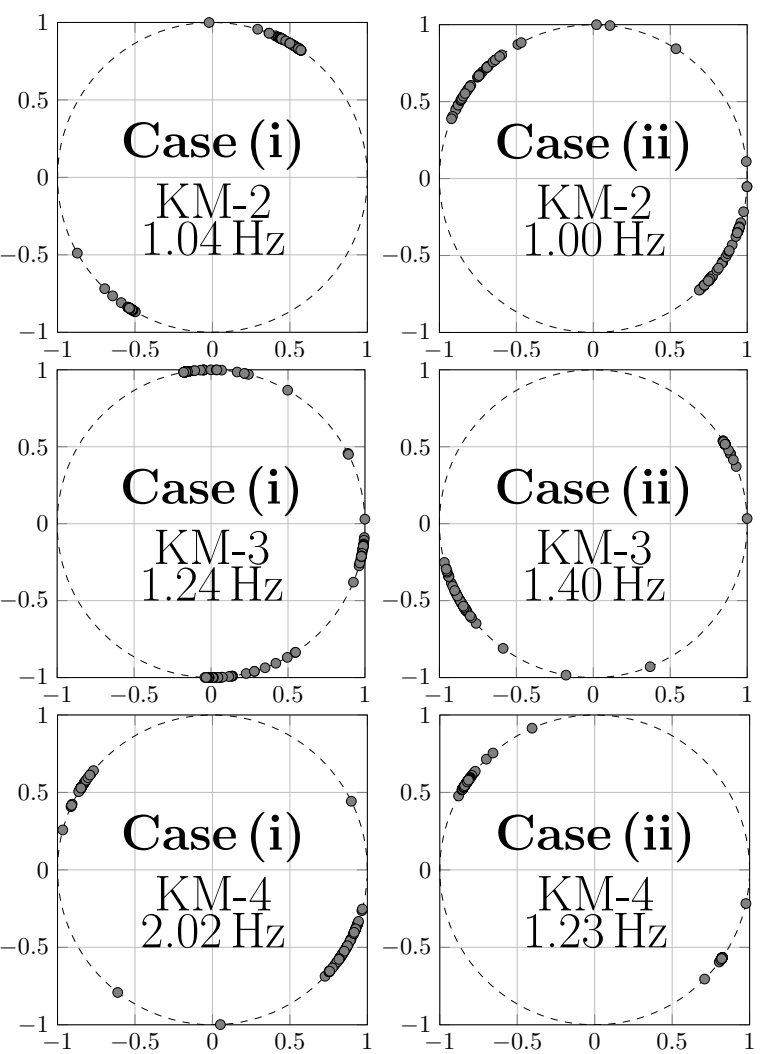

Figure 8: Initial phase vectors $\boldsymbol{\alpha}_{j}$ of selected oscillatory KMs listed in Table 1.

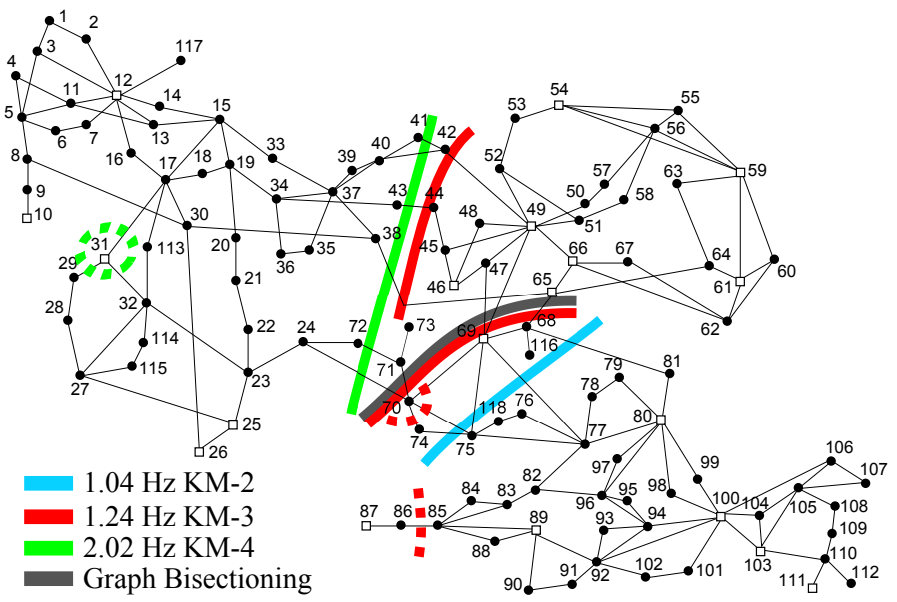

Figure 9: (Color online) Partitioning of the IEEE 118-bus test system based on phase-coherency in KMs for fault Case (i) (bus 17) and spectral graph bisectioning using $\boldsymbol{V}_{2}$. The cutsets are indicated by colored lines.

in [6] which extends the method in [32] to include load buses and not only to group generators behind transient reactances. The resulting 4-way partitioning for the slowcoherency based method is depicted in Fig. 10. As pointed in Figs. 9 and 10, the KMA-based partitioning identifies generator bus 87 as incoherent with the adjacent buses. In agreement with this, the slow-coherency-based method 


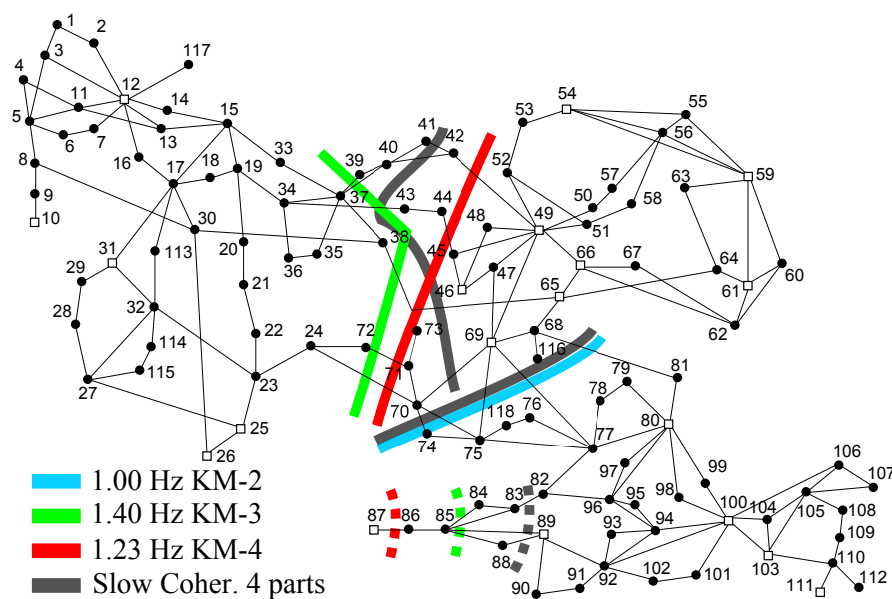

Figure 10: (Color online) Partitioning of the IEEE 118-bus test system based on phase-coherency in KMs for fault Case (ii) (bus 100) and a slow-coherency technique including load buses. The cutsets are indicated by colored lines.

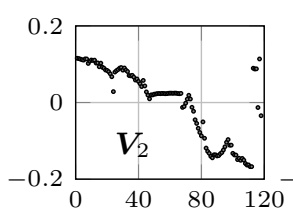

Bus Number

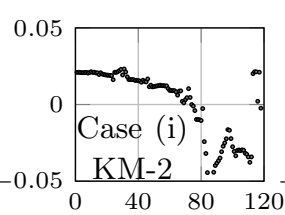

Bus Number

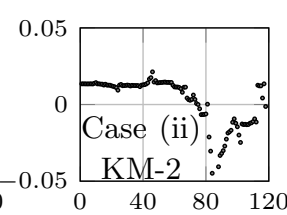

Bus Number
Figure 11: Comparison between the second eigenvector $\boldsymbol{V}_{2}$ of the graph Laplacian and spatial shapes $\boldsymbol{S}_{\mathrm{CC}}$ of KM-2 for both disturbance cases.

identifies bus 87 as an incoherent bus with respect to the adjacent buses. A more refined slow-coherency-based partitioning (not shown) reveals a new partition around bus 31 , as was identified by KM-4 for Case (i). On the other hand, graph theoretical methods do not take into account the peculiar behavior and interaction of dynamical components in the large test system. Additionally, KM-2's partition for Case (ii) is identical to one of the cutsets obtained from the slow-coherency technique. Also, KM-2 and 3 from Case (i) as well as spectral graph bisectioning provides a similar cutset. In fact, the partitioning obtained from KM-3 (1.24 $\mathrm{Hz}$ ) for Case (i) corresponds to a large extent to the 4-way partitioning using the slow-coherency technique. The same result is also achievable by combining the cutsets of two or more KMs.

\subsection{Advantages and Disadvantages}

The advantage of the proposed method lies in that it only requires a window of sampled dynamics and implicitly takes into account versatile features in the power system dynamics that otherwise requires a substantial amount of model parameters. With the expected increase in availability of synchronized measurements in the future, it is important to investigate new tools for monitoring and control based on
Table 2: Koopman modes obtained for the Case (ii) fault for different window lengths where $\Delta l_{\mathrm{w}}$ is the difference in number of samples from the original window length 421 . The modes that closely correspond to the previously identified KMs 2-4 have been picked up among the dominant modes.

\begin{tabular}{ccccccc}
\hline Case & \multicolumn{2}{c}{ 'KM-2' } & \multicolumn{2}{c}{ 'KM-3' } & \multicolumn{2}{c}{ 'KM-4' } \\
\hline$\Delta l_{\mathrm{w}}$ & Freq.[Hz] & Norm & Freq.[Hz] & Norm & Freq.[Hz] & Norm \\
\hline-30 & 1.07 & 0.30 & 1.37 & 0.29 & 1.25 & 0.71 \\
-6 & 1.10 & 0.36 & 1.32 & 0.38 & - & - \\
0 & 1.00 & 0.17 & 1.40 & 0.33 & 1.23 & 1.73 \\
6 & 0.96 & 0.14 & 1.38 & 0.73 & 1.18 & 0.82 \\
30 & 0.92 & 0.25 & 1.36 & 0.91 & 1.19 & 0.50 \\
\hline
\end{tabular}

sampled data. Since the proposed method is intended only for application to sampled data following large faults, it is limited to the extraction of the dynamic behavior excited by the disturbance, and should act as a complimentary tool to the standard power system analysis and control. For real-world applications of data-based methods, one has to be careful about noise in the data acquisition. It has been shown that the type of algorithm applied here is sensitive to noise [33], and thus care should be taken by filtering out the noise in the measured data.

\subsection{Computational Considerations}

Another important aspect of this method is the window length of the acquired data. Here, it is briefly demonstrated by a numerical example using the data on bus-angle swings shown in Fig. 7 (b). For the original window length in terms of samples; $l_{\mathrm{w}}=N+1=421$, we examine the results of KMA with the slightly perturbed window length $l_{\mathrm{w}}^{\prime}=l_{\mathrm{w}}+\Delta l_{\mathrm{w}}$ with $\Delta l_{\mathrm{w}}$ set to \pm 6 and \pm 30 (here, with our used $f_{\mathrm{s}}, \Delta l_{\mathrm{w}}=30$ corresponds to $0.5 \mathrm{~s}$ ). Note that the "starting point" of the window is the same for all window sizes. In Table 2, KMs are listed which closely correspond to the KMs previously used for the partitioning. In one case (for $\Delta l_{\mathrm{w}}=-6$ ), a KM close to 'KM-4' could not be picked up which is marked by '-' in the table. To exemplify how the partitioning result can change with varied window length, partitioning for 'KM-3' is given in Fig. 12 for all window lengths. It can be concluded that slightly different results can be obtained with different window lengths, but the results remain intact to a large extent despite perturbations of the window size. To achieve a reliable result it could be of importance to use a moving window and extract the result using a collection of measurements sets.

Finally, it deserves mentioning that the computation time for the KMA-algorithm (probably the most timeconsuming computation in the proposed method) using unoptimized code in MATLAB running on Windows 8 with a regular laptop PC (Intel Core i5-3317U $1.7 \mathrm{GHz}$ processor and $8 \mathrm{~GB}$ ram of RAM) is about $1.5 \mathrm{~s}$ and should not pose a problem for almost in real-time implementation and execution. 


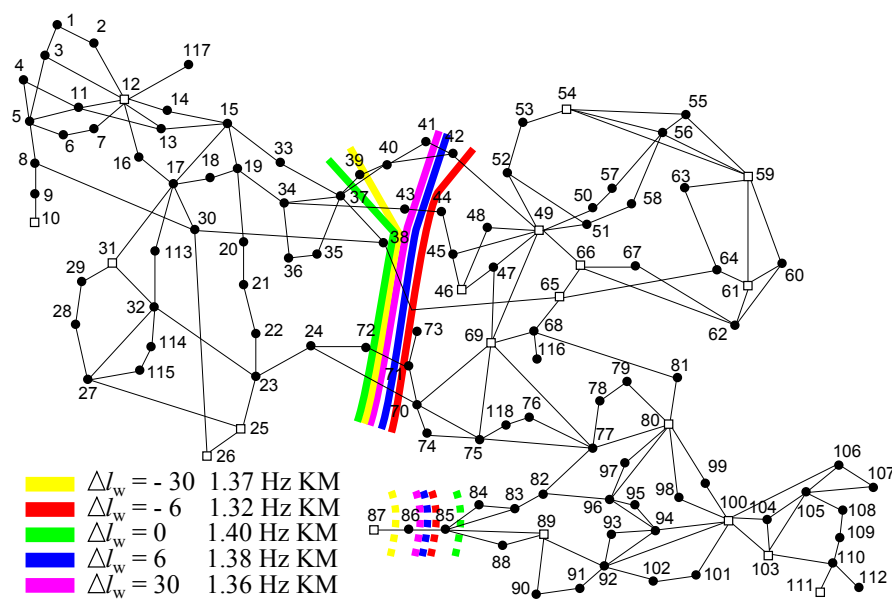

Figure 12: (Color online) Partitioning of the IEEE 118-bus test system for all cases in Table 2 for 'KM-3'.

\section{Conclusions}

In this paper we demonstrated a data-driven method for power network partitioning based on KMA. It was numerically and analytically shown that the data-driven method can cover network partitions derived from both spectral graph and a slow-coherency-based method. Apart from identifying oscillatory modal structures used for partitioning, the KMA-based partitioning pins down the frequency of oscillations as well as information on damping and participation, thus it can be used for monitoring and control purposes, namely the controlled islanding technique $[2,3]$.

Integrating the data-driven partitioning with a controlled islanding strategy is one of the future works. Such a realization could possibly incorporate PMU-based out-of-step protection as proposed in [34]. Further investigations for practical implementation of the proposed algorithm are of great importance: influence of network delay and measurement noise on the partitioning result, and data-acquisition methods for successful partitioning, e.g. improving the sample-mean method in the current observation of bus voltage angles.

\section{Acknowledgments}

The first author would like to thank Mr. H. R. Chamorro and Prof. M. Ghandhari (KTH Royal Institute of Technology) for useful discussions and suggestions during his time at KTH. The authors are also grateful to Prof. I. Mezić (UC Santa Barbara) for his suggestive comments on this work. Lastly, the authors would also like to thank anonymous reviewers for their valuable comments and suggestions.

\section{References}

[1] G. Andersson, P. Donalek, R. Farmer, N. Hatziargyriou, I. Kamwa, P. Kundur, N. Martins,
J. Paserba, P. Pourbeik, J. Sanchez-Gasca, R. Schulz, A. Stankovic, C. Taylor, and V. Vittal, "Causes of the 2003 major grid blackouts in north america europe, and recommended means to improve system dynamic performance," IEEE Transactions on Power Systems, vol. 20, no. 4, pp. 1922-1928, 2005.

[2] H. You, V. Vittal, and Z. Yang, "Self-healing in power systems: an approach using islanding and rate of frequency decline-based load shedding," IEEE Transactions on Power Systems, vol. 18, no. 1, pp. 174-181, Feb 2003.

[3] H. You, V. Vittal, and X. Wang, "Slow coherencybased islanding," IEEE Transactions on Power Systems, vol. 19, no. 1, pp. 483-491, 2004.

[4] B. Yang, V. Vittal, and G. T. Heydt, "Slow-coherencybased controlled islanding - A demonstration of the approach on the august 14, 2003 blackout scenario," IEEE Transactions on Power Systems, vol. 21, no. 4, pp. 1840-1847, 2006.

[5] J. H. Chow, R. Galarza, P. Accari, and W. W. Price, "Inertial and slow coherency aggregation algorithms for power system dynamic model reduction," IEEE Transactions on Power Systems, vol. 10, no. 2, pp. 680-685, 1995.

[6] S. Yusof, G. Rogers, and R. Alden, "Slow coherency based network partitioning including load buses," IEEE Transactions on Power Systems, vol. 8, no. 3, pp. 1375-1381, 1993.

[7] K. Sun, D. . Zheng, and Q. Lu, "Splitting strategies for islanding operation of large-scale power systems using OBDD-based methods," IEEE Transactions on Power Systems, vol. 18, no. 2, pp. 912-923, 2003.

[8] — , "A simulation study of OBDD-based proper splitting strategies for power systems under consideration of transient stability," IEEE Transactions on Power Systems, vol. 20, no. 1, pp. 389-399, 2005.

[9] K. Sun, D.-Z. Zheng, and Q. Lu, "Searching for feasible splitting strategies of controlled system islanding," IEE Proceedings: Generation, Transmission and Distribution, vol. 153, no. 1, pp. 89-98, 2006.

[10] L. Ding, F. Gonzalez-Longatt, P. Wall, and V. Terzija, "Two-step spectral clustering controlled islanding algorithm," IEEE Transactions on Power Systems, vol. 28 , no. 1 , pp. $75-84,2013$.

[11] R. Sanchez-Garcia, M. Fennelly, S. Norris, N. Wright, G. Niblo, J. Brodzki, and J. Bialek, "Hierarchical spectral clustering of power grids," IEEE Transactions on Power Systems, 2014.

[12] I. Mezić, "Spectral properties of dynamical systems, model reduction and decompositions," Nonlinear Dynamics, vol. 41, no. 1-3, pp. 309-325, 2005. 
[13] C. W. Rowley, I. Mezić, S. Bagheri, P. Schlatter, and D. S. Henningson, "Spectral analysis of nonlinear flows," Journal of Fluid Mechanics, vol. 641, pp. 115-127, 2009.

[14] M. Budišić, R. Mohr, and I. Mezić, "Applied Koopmanism," Chaos, vol. 22, no. 4, 2012.

[15] I. Mezić, "Analysis of fluid flows via spectral properties of the Koopman operator," Annual Review of Fluid Mechanics, vol. 45, pp. 357-378, 2013.

[16] Y. Susuki and I. Mezić, "Nonlinear Koopman modes and coherency identification of coupled swing dynamics," IEEE Transactions on Power Systems, vol. 26, no. 4, pp. 1894-1904, 2011, also, correction, this journal, vol. 26, no. 4, p. 2584, 2011.

[17] _ - "Nonlinear Koopman modes and a precursor to power system swing instabilities," IEEE Transactions on Power Systems, vol. 27, no. 3, pp. 1182-1191, 2012.

[18] _ - "Nonlinear Koopman modes and power system stability assessment without models," IEEE Transactions on Power Systems, vol. 29, no. 2, pp. 899-907, March 2014.

[19] F. Raak, Y. Susuki, T. Hikihara, H. R. Chamorro, and M. Ghandhari, "Partitioning power grids via nonlinear Koopman mode analysis," in Innovative Smart Grid Technologies Conference (ISGT), 2014 IEEE PES, Feb 2014, pp. 1-5.

[20] B. O. Koopman, "Hamiltonian systems and transformation in hilbert space," Proceedings of the National Academy of Sciences of the United States of America, vol. 17 , no. 5, p. 315, 1931.

[21] I. Kamwa, R. Grondin, and Y. Hébert, "Wide-area measurement based stabilizing control of large power systems - A decentralized/hierarchical approach," IEEE Transactions on Power Systems, vol. 16, no. 1, pp. 136-153, 2001.

[22] T. Bıyıkoğlu, J. Leydold, and P. Stadler, Laplacian Eigenvectors of Graphs: Perron-Frobenius and FaberKrahn Type Theorems, ser. Lecture notes in mathematics. Springer, 2007.

[23] M. Fiedler, "Algebraic connectivity of graphs," Czechoslovak Mathematical Journal, vol. 23, no. 98, pp. 298-305, 1973.

[24] — - "A property of eigenvectors of nonnegative symmetric matrices and its application to graph theory," Czechoslovak Mathematical Journal, vol. 25, pp. 619633, 1975.

[25] Y.-F. Huang, S. Werner, J. Huang, N. Kashyap, and V. Gupta, "State estimation in electric power grids: Meeting new challenges presented by the requirements of the future grid," IEEE Signal Processing Magazine, vol. 29, no. 5, pp. 33-43, 2012.

[26] J. De La Ree, V. Centeno, J. S. Thorp, and A. G. Phadke, "Synchronized phasor measurement applications in power systems," IEEE Transactions on Smart Grid, vol. 1, no. 1, pp. 20-27, 2010.

[27] T. Kanungo, D. M. Mount, N. S. Netanyahu, C. D. Piatko, R. Silverman, and A. Y. Wu, "An efficient kmeans clustering algorithms: Analysis and implementation," IEEE Transactions on Pattern Analysis and Machine Intelligence, vol. 24, no. 7, pp. 881-892, 2002.

[28] Power Systems Test Case Archive, College of Engineering, University of Washington. [Online]. Available: http://www.ee.washington.edu/research/pstca/.

[29] F. Milano, "An open source power system analysis toolbox," IEEE Transactions on Power Systems, vol. 20, no. 3, pp. 1199-1206, 2005.

[30] P. Kundur, Power System Stability and Control. McGraw-Hill, 1994.

[31] H.-D. Chiang and L. Fekih-Ahmed, "On the direct method for transient stability analysis of power system structure preserving models," in Circuits and Systems, 1992. ISCAS '92. Proceedings., 1992 IEEE International Symposium on, vol. 5, May 1992, pp. 2545-2548 vol.5.

[32] J. R. Winkelman, J. Chow, B. C. Bowler, B. Avramovic, and P. Kokotovic, "An analysis of interarea dynamics of multi-machine systems," IEEE Transactions on Power Apparatus and Systems, vol. PAS-100, no. 2, pp. 754-763, Feb 1981.

[33] D. Duke, J. Soria, and D. Honnery, "An error analysis of the dynamic mode decomposition," Experiments in Fluids, vol. 52, no. 2, pp. 529-542, 2012.

[34] Y. Deng, H. Lin, S. Shukla, J. Thorp, and L. Mili, "Cosimulating power systems and communication network for accurate modeling and simulation of pmu based wide area measurement systems using a global event scheduling technique," in Modeling and Simulation of Cyber-Physical Energy Systems (MSCPES), 2013 Workshop on, May 2013, pp. 1-6. 